\title{
Magnetic field dependence of conductivity and effective mass of carriers in a model of Mott-Hubbard material
}

\author{
L.Didukh; O.Kramar, Yu.Skorenkyy, Yu.Dovhopyaty \\ Ternopil State Technical University, \\ Department of Physics, \\ 56 Rus'ka Str., 46001 Ternopil, Ukraine
}

Received July 22, 2005

\begin{abstract}
The effect of external magnetic field $h$ on a static conductivity of MottHubbard material which is described by the model with correlated hopping of electrons has been investigated. By means of canonical transformation, the effective Hamiltonian is obtained which takes into account strong intra-site Coulomb repulsion and correlated hopping. Using a variant of generalized Hartree-Fock approximation the single-electron Green function and quasiparticle energy spectrum of the model have been calculated. The static conductivity $\sigma$ has been calculated as a function of $h$, electron concentration $n$ and temperature $T$. The correlated hopping is shown to cause the electron-hole asymmetry of transport properties of narrow band materials.
\end{abstract}

Key words: Mott-Hubbard material, conductivity, magnetic field

PACS: 72.15.-v, 72.80.Ga

The achievements of the recent years in the field of strongly correlated electron systems enable us to understand the properties of narrow-band materials, in particular those in which metal-insulator transition under the action of external effects (pressure, doping, temperature) is realized [1]. The strongly correlated electron systems demonstrate unusual transport properties [2]. In order to understand the physical mechanisms, which cause these peculiarities, the experimental and theoretical research of the temperature dependence of conductivity is needed. The results concerning the low-frequency behavior of conductivity are of prior importance, because they provide the information about the scattering processes close to the Fermi surface. The theoretical investigations of conductivity $\sigma(\omega, T)$ are mainly concentrated

*E-mail: didukh@tu.edu.te.ua 
on the limit $T=0$. The behavior of the static conductivity $\sigma(T)=\sigma(\omega=0, T)$ at $T>0$ has not been studied well enough.

Theoretical investigations of the optical conductivity in the Hubbard model [3] within the framework of the Kubo linear response theory [4] have been going on for many decades. Here we should note the investigations by analytical methods such as: moment method [5], composite operators method [6], the mean-field theory $[7,8]$, the perturbative theory method $[9,10]$ in the limit of weak interaction $(|t| \gg U)$, the method of the memory function $[11,12]$ in the opposite limit $(U \gg|t|)$. The conductivity has been intensively studied in the one-dimensional Hubbard model [13-15], where the numerical results can be compared with the exact ones obtained by Bethe ansatz application.

In order to numerically investigate the conductivity in the Hubbard model, the exact diagonalization of finite clusters has been used [16] for $4 \times 4$ sites cluster, quantum Monte-Carlo method for 8, 10 sites [17], 3 x 3 sites [15], 8 x 8 sites [18], 12 x 12 sites [19]. The investigations using numerical methods were mainly carried out for a two-dimensional lattice (this is caused by the interest to the high-temperature superconductivity phenomena [20] in the systems with $\mathrm{CuO}$ planes). The conductivity of three-dimensional system has been studied only in a narrow interval close to half-filling at weak and intermediate interactions [21].

The optical conductivity has also been studied using the dynamical mean field theory (DMFT) in the limit of infinite spatial dimension [22]. Different DMFT equations solvers were used: iterated perturbation theory IPT [23], non-crossing approximation NCA [24], second order perturbation theory 2OPT [25]. The authors have considered the symmetrical Hubbard model at half-filling close to metal-insulator transition (intra-atomic Coulomb repulsion $U \simeq 2 w$, where $2 w$ is the bandwidth) and have obtained a good agreement with experimental data for some Mott-Hubbard systems. However, for realistic models the non-local (i.e., dependent on wave vector) contributions to self-energy and transport characteristics are important. Besides, the investigations within the framework of DMFT consider mainly the case of half-band filling.

In works [26-28] the essential importance of taking into account the correlated hopping has been emphasized and narrow-band model with non-equivalent Hubbard subbands has been proposed. In such a model the hopping integrals which describe the translational movement of holes and doublons differ from one another and from the activation processes integral. Similar models have been studied intensively in recent years [29-31]. For the generalized model which takes into account the nonequivalency of Hubbard subbands, there are no reliable results for conductivity, so the analytical study of conductivity within the framework of realistic models of electronic subsystem is necessary.

In this work we show that the application of the variant of projection procedure for the calculation of the Green function allows us to reproduce some peculiarities of static conductivity of narrow-band material in the limit of strong Coulomb correlation and to investigate the external effects such as temperature change, doping, pressure and magnetic field. We apply our approach to the Hamiltonian, which, besi- 
des the intra-site Coulomb repulsion $U$, strong in comparison with inter-site hopping $t_{i j}$, describes the correlated hopping of electrons (the effect of electron concentration $n$ on the hopping processes) and show that it leads to the electron-hole asymmetry of conductivity and other characteristics.

We write the Hamiltonian of the correlated electron system in representation of $X_{i}^{k l}$ Hubbard operators:

$$
H=H_{0}+H_{1}+H_{1}^{\prime}+H_{\mathrm{ex}}
$$

where

$$
\begin{aligned}
& H_{0}=-\mu \sum_{i s}\left(X_{i}^{s}+X_{i}^{2}\right)+U \sum_{i} X_{i}^{2}+\frac{1}{2} N V_{0} \kappa u^{2}+\mu_{\mathrm{B}} h \sum_{i s} \eta_{s} X_{i}^{s}, \\
& H_{1}=\sum_{i j s}^{\prime} t_{i j}(n) X_{i}^{s 0} X_{j}^{0 s}+\sum_{i j s}^{\prime} \tilde{t}_{i j}(n) X_{i}^{2 s} X_{j}^{s 2}, \\
& H_{1}^{\prime}=\sum_{i j s}^{\prime}\left(t_{i j}^{\prime}(n) \eta_{s} X_{i}^{s 0} X_{j}^{\bar{s} 2}+h . c .\right) . \\
& H_{\mathrm{ex}}=-\frac{1}{2} \sum_{i j s}^{\prime} J(i j)\left(\left(X_{i}^{s}+X_{i}^{2}\right)\left(X_{j}^{s}+X_{j}^{2}\right)+X_{i}^{s \bar{s}} X_{j}^{\bar{s} s}\right) .
\end{aligned}
$$

Here operator $X_{i}^{k l}$ describes the transition of site $i$ from state $|l\rangle$ to state $|k\rangle, \mu$ is the chemical potential, $U$ denotes the energy of intra-site Coulomb repulsion of electrons, $J$ stands for the direct inter-site exchange interaction, $\kappa$ is the elastic constant, $V_{0}$ is the initial volume of the crystal, $N$ is the number of lattice sites, $\mu_{\mathrm{B}}$ is Bohr magneton, $h$ stands for the external magnetic field, $\eta_{\mathrm{s}}=1$ if $s=\uparrow$ and -1 otherwise. Translation processes are characterized by different hopping integrals, namely $t_{i j}(n)=$ $(1+\alpha u)\left(1-\tau_{1} n\right) t_{i j}$ and $\tilde{t}_{i j}(n)=(1+\alpha u)\left(1-\tau_{1} n-2 \tau_{2}\right) t_{i j}$ are hopping parameters for holes and doublons, respectively; $t_{i j}^{\prime}(n)=(1+\alpha u)\left(1-\tau_{1} n-\tau_{2}\right) t_{i j}$ is parameter of the hopping of an electron between doublon and hole; correlated hopping parameters $\tau_{2}$ and $\tau_{1}$ describe the effect of the sites involved in the hopping process and neighboring sites, respectively; parameter $\alpha<0$ takes into account the renormalization of bandwidth $2 w=2 z\left|t_{i j}\right|$ at strain $u$ [32], $z$ is the number of the nearest neighbor to a site.

We restrict ourselves to considering the strong correlation limit, namely $U \gg$ $w(n)$. In such a system at partial filling of the band the conductance is mainly due to electron hopping processes within the Hubbard subbands. Thus, the interband hopping can be neglected. At these conditions we apply the canonical transformation [28] to the Hamiltonian (1).

$$
H_{\text {eff }}=\mathrm{e}^{S} H \mathrm{e}^{-S},
$$

where

$$
S=\sum_{i j}\left(L(i j)\left(X_{i}^{\uparrow 0} X_{j}^{\downarrow 2}-X_{j}^{\downarrow 0} X_{i}^{\uparrow 2}\right)-\text { h.c. }\right)
$$


with $L(i j)=t_{i j}^{\prime}(n) / U$. The operator $S$ is taken to exclude the processes with pair hopping of holes and doublons in the first order in the hopping parameter:

$$
H_{1}^{\prime}+\left[S ; H_{0}\right]=0 .
$$

Finally, we obtain the effective Hamiltonian:

$$
H_{\mathrm{eff}}=H_{0}+H_{1}+H_{\mathrm{ex}}+\tilde{H}_{\mathrm{ex}}
$$

where

$$
\tilde{H}_{\mathrm{ex}}=-\frac{1}{2} \sum_{i j s}^{\prime} \tilde{J}(i j)\left(X_{i}^{s} X_{j}^{\bar{s}}-X_{i}^{s \bar{s}} X_{j}^{\bar{s} s}\right)
$$

with the indirect exchange interaction parameter $\tilde{J}(i j)=\left(t_{i j}^{\prime}(n)\right)^{2} / U$.

Using the projection procedure [33] for the case of $n<1$ we obtain for the single particle energy spectrum:

$$
E_{\mathrm{s}}(\mathbf{k})=-\mu-z J n_{\mathrm{s}}-z \tilde{J} n_{\overline{\mathrm{s}}}+\alpha_{\mathrm{s}} t_{\mathbf{k}}(n)+\beta_{\mathrm{s}}
$$

where the correlated narrowing of the band and spin-dependent shift of subband center are

$$
\begin{aligned}
\alpha_{\mathrm{s}} & =\frac{2-n+\eta_{\mathrm{s}} m}{2}+\frac{n^{2}-m^{2}}{2\left(2-n+\eta_{\mathrm{s}} m\right)} \\
\beta_{\mathrm{s}} & =-\frac{2}{\left(2-n+\eta_{\mathrm{s}} m\right)} \sum_{\mathbf{k}} t_{\mathbf{k}}(n)\left\langle X_{i}^{\bar{s} 0} X_{j}^{0 \bar{s}}\right\rangle_{\mathbf{k}}
\end{aligned}
$$

respectively. The respective results for $n>1$ :

$$
\begin{aligned}
\tilde{E}_{\mathrm{s}}(\mathbf{k}) & =-\mu+U-z J n_{\mathrm{s}}-z \tilde{J} n_{\overline{\mathrm{s}}}+\tilde{\alpha}_{\mathrm{s}} \tilde{t}_{\mathbf{k}}(n)+\tilde{\beta}_{\mathrm{s}} \\
\tilde{\alpha}_{\mathrm{s}} & =\frac{n+\eta_{\mathrm{s}} m}{2}+\frac{n^{2}-m^{2}}{2\left(n+\eta_{\mathrm{s}} m\right)} \\
\tilde{\beta}_{\mathrm{s}} & =-\frac{2}{\left(n+\eta_{\mathrm{s}} m\right)} \sum_{\mathbf{k}} \tilde{t}_{\mathbf{k}}(n)\left\langle X_{i}^{s 2} X_{j}^{2 s}\right\rangle_{\mathbf{k}}
\end{aligned}
$$

describe the upper Hubbard subband of halfbandwidth $\tilde{w}(n)=z\left|\tilde{t}_{i j}(n)\right|$.

Using the method of works $[34,35]$, we calculate the $x x$-component of static electronic conductivity $\sigma_{x x}=\sigma+\tilde{\sigma}$, where

$$
\sigma=-\frac{\mathrm{e}^{2} \tau z}{2 N a} \sum_{i j s}\left\langle X_{i}^{s 0} X_{j}^{0 s}\right\rangle t_{i j}(n),
$$

is the conductivity of lower $(0-s)$-subband,

$$
\tilde{\sigma}=-\frac{\mathrm{e}^{2} \tau z}{2 N a} \sum_{i j s}\left\langle X_{i}^{2 s} X_{j}^{s 2}\right\rangle \tilde{t}_{i j}(n),
$$




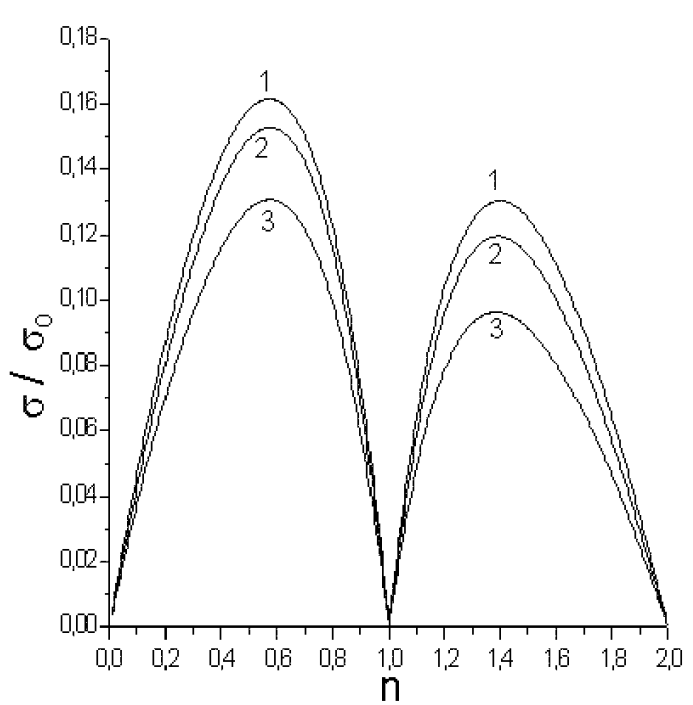

Figure 1. The concentration dependencies of the conductivity at correlated hopping $\tau_{1}=\tau_{2}=0.1$ in the absence of the external magnetic field and exchange interactions: curve 1 corresponds to temperature $\Theta / w=0.01$, curve 2 corresponds to $\Theta / w=0.1$, curve 3 corresponds to $\Theta / w=0.2$.

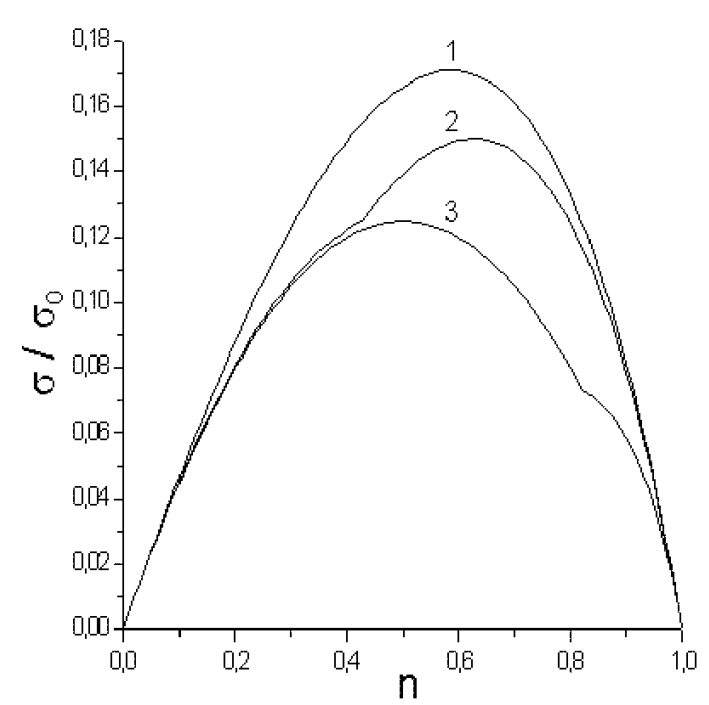

Figure 2. The concentration dependencies of the conductivity in the external magnetic field at $\Theta / w=0.02$, $z J_{\text {eff }} / w=0.02, \tau_{1}=\tau_{2}=0$ : curve 1 corresponds to $h / w=0$, curve 2 corresponds to $h / w=0.005$, curve 3 corresponds to $h / w=0.02$.

is the conductivity of upper $(\uparrow \downarrow-\bar{s})$-subband.

The magnetization can be calculated from the equation

$$
\exp \left(\frac{2 h+\beta_{\downarrow}-\beta_{\uparrow}+z J_{\mathrm{eff}} m}{\Theta}\right)=\frac{\sinh \left(\frac{w(n) \alpha_{\downarrow}}{\Theta} \frac{1-n}{1-n_{\uparrow}}\right)}{\sinh \left(\frac{w(n) \alpha_{\uparrow}}{\Theta} \frac{1-n}{1-n_{\downarrow}}\right)} \frac{\sinh \left(\frac{w(n) \alpha_{\uparrow}}{\Theta} \frac{n_{\uparrow}}{1-n_{\downarrow}}\right)}{\sinh \left(\frac{w(n) \alpha_{\downarrow}}{\Theta} \frac{n_{\downarrow}}{1-n_{\uparrow}}\right)}
$$

for $n<1$ and corresponding equation for $n>1$ in which the substitutions $n \rightarrow 2-n$, $w(n) \rightarrow \tilde{w}(n), \alpha_{\mathrm{s}} \rightarrow \tilde{\alpha}_{\mathrm{s}}, \beta_{\mathrm{s}} \rightarrow \tilde{\beta}_{\mathrm{s}}$ are made. Here $J_{\text {eff }}=J-\tilde{J}$. Using these expressions we have numerically calculated the static conductivity $\sigma_{x x}$ as a function of electron concentration (figures 1, 2), temperature (figure 4), and magnetic field (figure 3).

From figure 1 one can see that in the considered model with correlated hopping of electrons, the conductivity provided by the carriers in the upper subband is lower than the conductivity, provided by carriers in the lower subband. This effect was discussed in work [35]. This is a manifestation of the electron-hole asymmetry inherent to real transition metal compounds. Another important feature is the change of the current carrier type from metallic to semiconducting type in the vicinity of $n=2 / 3,4 / 3$ and from semiconducting type to the metallic one at $n=1$. Increasing the correlated hopping we shift the maxima of the conductivity closer to the half-filling. The external magnetic field qualitatively changes the concentration dependence of $\sigma$ (see figure 2). 


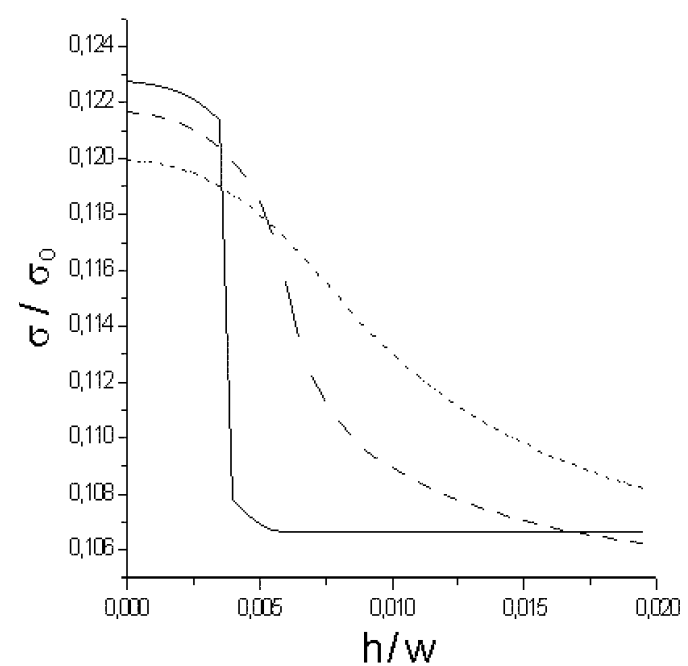

Figure 3. The dependencies of the conductivity on magnetic field for the electron concentration $n=0.3$ and $z J_{\text {eff }} / w=0.01, \tau_{1}=\tau_{2}=0$ : solid curve corresponds to $\Theta / w=0.02$, dashed curve - to $\Theta / w=0.04$, dashed-dotted curve - to $\Theta / w=0.06$.

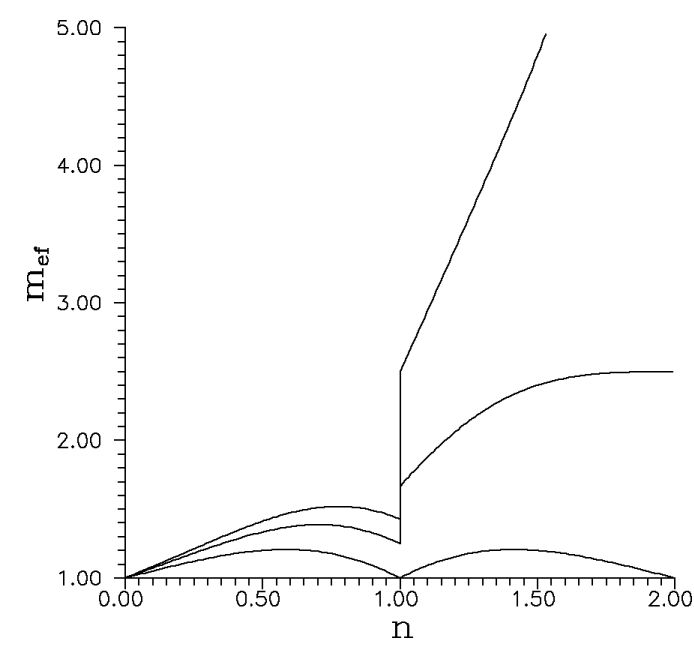

Figure 5. The dependence of current carriers effective mass $m_{\text {eff }} / m_{0}$ on electron concentration: upper curves correspond to $\tau_{1}=\tau_{2}=0.3$; middle curves - to $\tau_{1}=$ $\tau_{2}=0.2$; lower curves - to $\tau_{1}=\tau_{2}=0$.

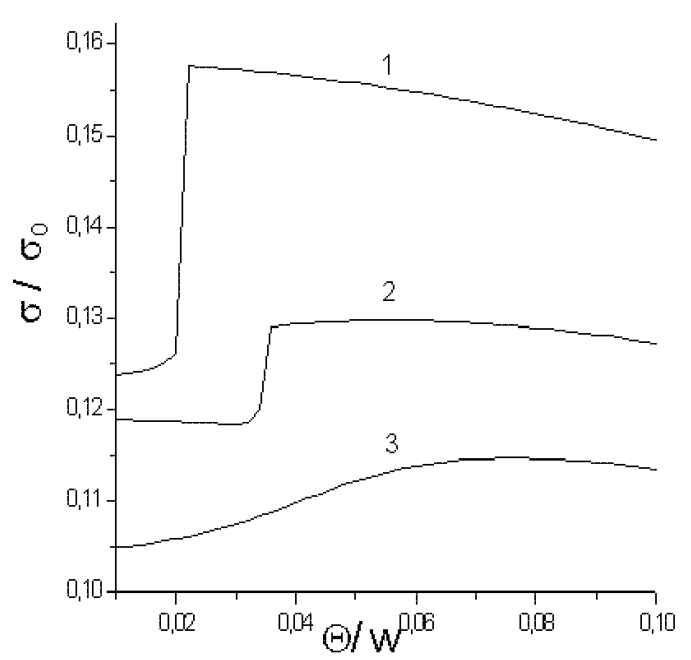

Figure 4. The dependencies of the conductivity on temperature in the external magnetic field $h / w=0.01$ at $z J_{\text {eff }} / w=0$ and $\tau_{1}=\tau_{2}=0$ : curve 1 corresponds to $n=0.3$, curve 2 - to $n=0.35$, curve $3-$ to $n=0.45$.

The higher is the electron concentration, the less pronounced is the effect of the applied magnetic field. At small concentration of electrons the band is fully polarized. In such a ferromagnetic system the conductivity is considerably lower than in paramagnetic state. If the electron concentration increases, the decrease of magnetization leads to the increase of conductivity, $\sigma$ approaches its value in the paramagnetic state. At the same time, due to the changes in the correlation band narrowing factor and the shift of subband center, the position of conductivity maximum changes from $n=2 / 3$ in the saturated ferromagnetic state (figure 2,2) to $n=0.5$ in the ferromagnetic state (figure 2,3 ). The change of conductivity with the increase of the applied field can be very sharp at low temperature, (figure 3, solid curve). The increase of temperature leads to the decrease of $\sigma$ 


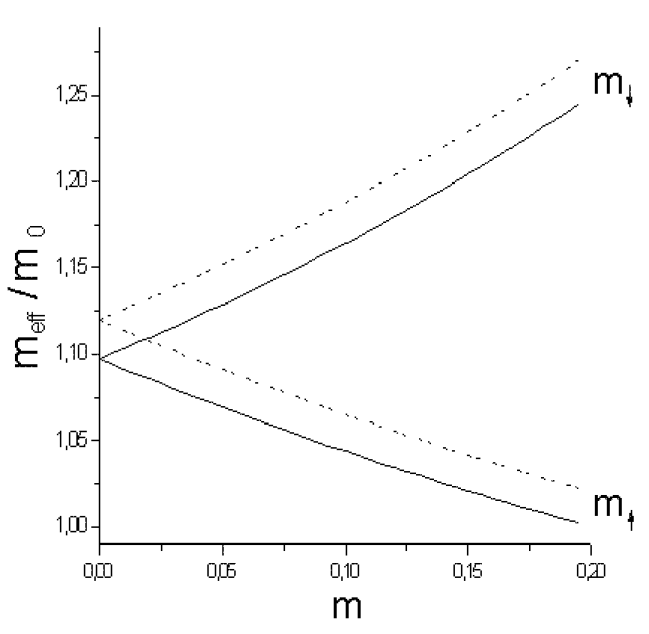

Figure 6. The dependence of current carriers effective mass $m_{\mathrm{eff}} / m_{0}$ on the magnetization at $n=0.2, \Theta / w=0.02$, $z J_{\text {eff }} / w=0$ : solid curves correspond to $\tau_{1}=\tau_{2}=0$; dashed curves - to $\tau_{1}=$ $\tau_{2}=0.1$.

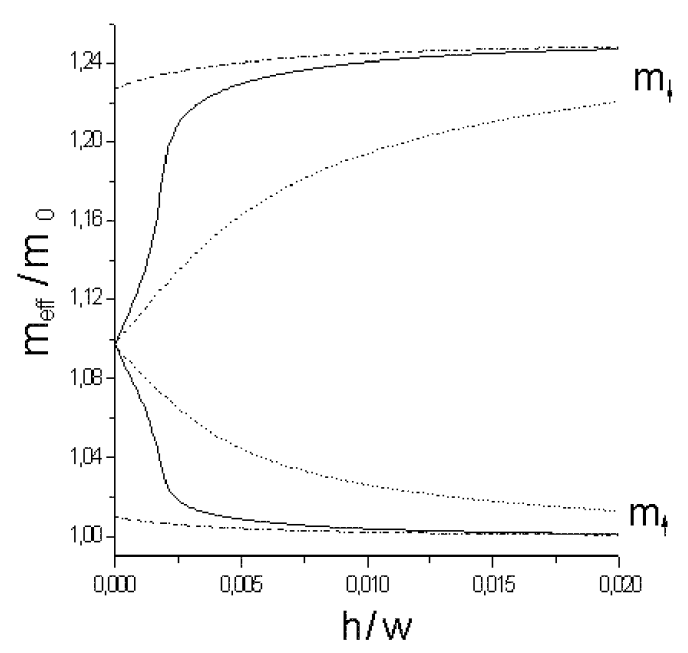

Figure 7. The dependence of current carriers effective mass $m_{\mathrm{eff}} / m_{0}$ on the applied magnetic field: solid curves correspond to $z J_{\text {eff }} / w=0, \Theta / w=0.02$; dotted curves - to $z J_{\text {eff }} / w=0, \Theta / w=$ 0.05 ; dash-dotted curves - to $z J_{\text {eff }} / w=$ $0.05, \Theta / w=0.02$.

and its changes become more smooth. Plateau of $\sigma(h)$ dependence signifies that at low temperatures a fully polarized state is reached in a very low field. Much higher field is needed to polarize spins at higher temperatures (figure 3, long-dashed and short-dashed curves).

Sharp increase of $\sigma$ with the increase of temperature is related to the transition from the polarized ferromagnetic state to the paramagnetic one. In each state the conductivity decreases with the rise of temperature. Very small changes of electron concentration can greatly affect the temperature dependence of conductivity (see figure 4).

Within the framework of the considered model one can naturally introduce the notions of "wide" (lower) and "narrow" (upper) energy bands and, correspondingly, "light" and "heavy" current carriers with the effective masses

$$
m_{\mathrm{s}}^{*}=\left(\frac{\partial^{2} E_{\mathrm{s}}(\mathbf{k})}{\partial \mathbf{k}^{\mathbf{2}}}\right)^{-1}, \quad \tilde{m}_{\mathrm{s}}^{*}=\left(\frac{\partial^{2} \tilde{E}_{\mathrm{s}}(\mathbf{k})}{\partial \mathbf{k}^{\mathbf{2}}}\right)^{-1},
$$

where $E_{\mathrm{s}}(\mathbf{k})$ is the energy spectrum of current carriers in the lower $(s-0)$ band, $\tilde{E}_{\mathrm{s}}(\mathbf{k})$ is the energy spectrum of current carriers in the upper $(\bar{s}-\uparrow \downarrow)$ subband.

We have found that the effective mass of heavy carriers can increase substantially with the increase of electron concentration. Due to the correlated hopping, the heavy carriers can be realized in the lower $(s-0)$ subband as well. It is worthwhile to note that the notion "effective mass of current carrier" has a conditional sense, different from the standard one, used in the band theory. The definitions (16) are related to the expressions for band spectrum which describe the transitions between $|s\rangle$ - and 
$|0\rangle$-states as well as $|\uparrow \downarrow\rangle$ - and $|\bar{s}\rangle$-states. In the paramagnetic state $m^{*}$ and $\tilde{m}^{*}$ are effective masses of respective transitions; for the cases when subbands are almost empty or almost full, $m^{*}$ and $\tilde{m}^{*}$ can be interpreted as effective masses of electronic and hole states. For the case $n \ll 1$ the value $m_{1}^{*}=\hbar\left(2 a^{2}|t(n)|\right)^{-1} \simeq \hbar\left(2 a^{2}|t|\right)^{-1}$ can be identified as an effective mass of $|s\rangle$-states, i.e. electrons, for the case $n=1-\varepsilon$ $(\varepsilon \ll 1)$ the value $m_{2}^{*}=\left.\hbar\left(2 a^{2}|t(n)|\right)^{-1}\right|_{n=1}$ is an effective mass of a hole. If $n>1$, then for $n=1+\varepsilon$ we have translational motion of doublons ("extra electrons") with masses $\tilde{m}_{1}^{*}=\left.\hbar\left(2 a^{2}|\tilde{t}(n)|\right)^{-1}\right|_{n=1}$, and for $n=2-\varepsilon$ we have the effective mass of holes $\tilde{m}_{2}^{*}=\left.\hbar\left(2 a^{2}|\tilde{t}(n)|\right)^{-1}\right|_{n=2}$. From these results one can see that the effective mass of carrier in the upper band can substantially differ from the effective masses in the case when the conductivity is due to $s-0$-transitions. It is important to note that passing from the regime of conductivity provided by the carriers in the lower band to the regime when it is provided by $\bar{s}-\uparrow \downarrow$-transitions, the effective mass increases stepwise at the point $n=1$ (the effective mass dependence on the electron concentration is shown in figure 5 ).

The effective mass dependencies on the magnetization and external magnetic field are shown in figures 6 and 7, respectively. The rise of magnetization leads to the rise of difference in the effective masses of spin-up and spin-down current carriers (figure 6). The slope of the dependencies $m_{\text {eff }}(m)$ changes with the rise of $m$ as well. This leads to the decrease of overall transport in ferromagnetic state, though the effective mass of carriers with majority spin becomes lower. The results shown in figure 6 qualitatively agree with the corresponding plot of work [36], where Gutzwiller approximation has been used to calculate effective masses of current carriers. The correlated hopping, favoring localization, shifts the effective masses up. Different possible scenarios of $m_{\text {eff }}(h)$ dependence are shown in figure 7 . At high temperature $m_{\text {eff }}$ changes monotonously (figure 7 , dotted curve) while at low temperature the system in unstable towards the transition to polarized state (figure 7 , solid curve). The direct exchange interaction can stabilize the ferromagnetically polarized state in the less than half-filled band even in a weak magnetic field (figure 7, dash-dotted curve).

In this paper we have used a model with correlated hopping of electrons to study the effect of the external magnetic field, temperature and doping on a static conductivity of Mott-Hubbard material. In the regime of strong Coulomb interaction hybridization of the Hubbard subbands does not contribute essentially to the transport properties, so we have excluded it from the effective Hamiltonian by means of canonical transformation. The single-electron Green function and quasiparticle energy spectrum of the model have been calculated using a variant of generalized Hartree-Fock approximation. This procedure has allowed us to obtain an analytical expression for the band narrowing factor and relatively simple and transparent equations for calculation of spin-dependent shift of the band center, magnetization, static conductivity and effective mass of the current carrier. The results of our study generalize the results of works $[6,16]$, where the static conductivity of the Hubbard model has been calculated as a function of electron concentration $n$, for a wider class of systems, for which the correlated hopping should be taken into account. In the limiting case of the absence of correlated hopping our results agree with the 
concentrational dependencies obtained in the composite operator method [6], exact diagonalization [16] and Monte-Carlo simulations [18]. The temperature dependence of conductivity in paramagnetic state, calculated in this work, agrees with the corresponding results of DMFT [24,37]. In the magnetic field, the static conductivity reflects the changes of single electron energy spectrum through correlation narrowing of the band and the shift of subband center. The temperature and concentration dependencies of $\sigma$ are governed by the changes of system magnetization in the external magnetic field. We have found that in the ground state the saturated ferromagnetic phase is stable while at non-zero temperature, the magnetization has the concentration dependence which agrees with work [38]. Such a behavior of magnetization leads to $\sigma(n)$ dependence with maxima at quarter and three-quarter fillings unlike in paramagnetic ones, obtained in work [35]. At non-zero temperatures the sharp changes of $\sigma(n)$ dependence are possible. It is due to the complicated character of temperature dependence of the band narrowing factor. The effective mass of quasiparticles appears to be spin-dependent and substantially varies with the magnetic field. These results are in agreement with the analysis of work [36] and with the experimental data of work [39] for heavy-fermion compounds. The correlated hopping inherent to real narrow band materials being taken into consideration enables us to describe electron-hole asymmetry of the processes observed in real materials.

\section{Acknowledgement}

Authors are grateful to Prof. I.V.Stasyuk, Prof. J.Spałek and Dr. A.M.Shvajka for the enlightening discussions. This work was supported by Ukrainian Fund for Fundamental Research under grant No. 02.07/266.

\section{References}

1. Imada M., Fujimori A., Tokura Y., Rev. Mod. Phys., 1998, 70, 1039.

2. Mott N.F. Metal-Insulator Transition. Taylor \& Francis, London, 1990.

3. Hubbard J., Proc. Roy. Soc., 1963, A276, 238.

4. Kubo R., J. Phys. Soc. Jpn., 1957, 12, 570.

5. Ohata N., Kubo R., J. Phys. Soc. Jpn., 1970, 28, 1402.

6. Mancini W., Villiani D., Phys. Let. A, 1999, 261, 357.

7. Hirsch J.E., Phys. Rev. B, 1999, 59, 6256.

8. Hirsch J.E., Phys. Rev. B, 2000, 62, 14131.

9. Brenig W., Z. Phys., 1992, 89, 187.

10. Wermbter S., Tewordt L., Physica C, 1993, 211, 132.

11. Goetze W., Woelfle P., Phys. Rev. B, 1972, 6, 1226.

12. Plakida N., J. Phys. Soc. Jpn., 1996, 65, 3964.

13. Fye R., Martins M., Scalettar R.T., Phys. Rev. B, 1990, 42, 6809.

14. Fye R. et al., Phys. Rev. B, 1991, 44, 6909.

15. Stephan W., Horsh P., Phys. Rev. B, 1990, 42, 8736.

16. Dagotto E., Rev. of Mod. Phys., 1994, 66, 763.

17. Moreo A., Dagotto E., Phys. Rev. B, 1990, 42, 4786. 
18. Scalapino D.J., White S.R., Zhang S.C., Phys. Rev. Lett., 1992, 68, 2830.

19. Bulut N., Scalapino D.J., White S.R., Phys. Rev. Lett., 1994, 73, 748.

20. Plakida N.M. High-Temperature Superconductivity. Springer-Verlag, Berlin, 1995.

21. Tan L., Callaway J., Phys. Rev. B, 1992, 46, 5499.

22. Georges A., Kotliar G., Krauth W., Rozenberg M., Rev. Mod. Phys., 1996, 68, 13.

23. Georges A., Krauth W., Phys. Rev. B, 1993, 48, 7167.

24. Pruschke Th., Cox D.L., Jarrel M., Europhys. Lett., 1993, 21, 593.

25. Rozenberg M., et al., Phys. Rev. Lett., 1995, 75, 105.

26. Didukh L. - In: Abstracts, Ukrainian-French Simposium "Condensed Matter: Science and Industry", Lviv, 1993, p. 275.

27. Didukh L. Preprint of Institute for Condensed Matter Physics, ICMP-96-20U, 32 p., Lviv, 1996 (in Ukrainian).

28. Didukh L., Journ. of Phys. Stud., 1997, 1, 241 (in Ukrainian).

29. Aligia A.A., Arrachea L., Gagliano E.R., Phys. Rev. B, 1995, 51, 13774.

30. Lin H.Q., Hirsch J.E., Phys. Rev. B, 1995, 52, 16155.

31. Gagliano E.R. et al., Phys. Rev. B, 1995, 51, 14012.

32. Grygorchuk R.A., Stasyuk I.V., Ukr. Phys. Journ., 1980, 25, 404 (in Russian).

33. Didukh L., Cond. Matter Phys., 1998, 1, 125.

34. Bari R.H., Adler D., Lange R.V., Phys. Rev. B, 1970, 2, 2898.

35. Didukh L. et al. Preprint of Institute for Condensed Matter Physics, ICMP-03-31U, 30 p., Lviv, 2003 (in Ukrainian).

36. Spałek J., Gopalan P., Phys. Rev. Lett., 1990, 64, 2823.

37. Uhrig G., Vollhardt D., Phys. Rev. B, 1995, 52, 5617.

38. Didukh L., Kramar O., Condens. Matter Phys., 2005, 8, No. 3(43), 547.

39. McCollam A. et al, Physica B, 2005, 359-361, 1-8.

\title{
Залежність провідності та ефективної маси носіїв від магнітного поля в моделі Мотт-Габбардівського матеріалу
}

\author{
Л.Дідух, О.Крамар, Ю.Скоренький, Ю.Довгоп'ятий \\ Тернопільський державний технічний \\ університет ім. І. Пулюя, кафедра фізики, \\ Україна, 46001 Тернопіль, вул. Руська, 56
}

Отримано 22 липня 2005 р.

В статті досліджено вплив зовнішнього магнітного поля на статичну провідність Мотт-Габбардівського матеріалу в моделі з корельованим переносом електронів. Ефективний гамільтоніан отримано із застосуванням канонічного перетворення та з використанням узагальненого наближення Гартрі-Фока знайдено функцію Гріна і енергетичний спектр. Провідність розрахована як функція магнітного поля, концентрації електронів та температури.

Ключові слова: Мотт-Габбардівський матеріал, провідність, магнітне поле

PACS: $72.15 .-v, 72.80 . G a$ 